\title{
STUDYING LINKS VIA CLOSED BRAIDS. V: THE UNLINK
}

\author{
JOAN S. BIRMAN AND WILLIAM W. MENASCO
}

\begin{abstract}
The main result is a version of Markov's Theorem which does not involve stabilization, in the special case of the $r$-component link. As a corollary, it is proved that the stabilization index of a closed braid representative of the unlink is at most 1 . To state the result, we need the concept of an "exchange move", which modifies a closed braid without changing its link type or its braid index. For generic closed braids exchange moves change conjugacy class. Theorem 1 shows that exchange moves are the only obstruction to reducing a closed $n$-braid representative of the $r$-component unlink to the standard closed $r$-braid representative, through a sequence of braids of nonincreasing braid index.
\end{abstract}

\section{INTRODUCTION}

Let $\boldsymbol{K}$ be an oriented link type in oriented $S^{3}, \mathbf{K}$ a representative of $\boldsymbol{K}$, and $\mathbf{A}$ an unknotted curve in $S^{3}$. $\mathbf{K}$ is a closed $n$-braid with braid axis $\mathbf{A}$ if $\mathbf{K}$ winds monotonically about $\mathbf{A}$ with total winding number $n$. "Monotonically" means that if $\mathrm{A}$ is the $z$-axis in $R^{3}$, parametrized by cylindrical coordinates $(r(t), \theta(t), z(t))$, then $\theta(t)$ is a strictly increasing function of $t$. This is equivalent to the assertion that if $\left\{\mathbf{H}_{t}: t \in[0,2 \pi]\right\}$ is a fibration of $S^{3}-\mathbf{A}$ by meridian discs then $\mathbf{K}$ meets each $\mathbf{H}_{t}$ transversally in exactly $n$ coherently oriented intersections. Alexander proved in [A] that every link could be so-represented (in many ways). The theorem which is known as Markov's theorem describes how the various closed braid representatives of a given link type are related. Markov's Theorem was announced in [Ma], with a working outline for a proof. It has been important in recent years because of its central role in the theory of knot polynomials [J]. The first complete proof was given in [Bi], using the methods suggested by Markov. Two other proofs have been given more recently in [Be, Mo,2].

Markov's Theorem. Let $\mathbf{K}$ be an arbitrary closed $n$-braid representatives of $\boldsymbol{K}$, and let $\mathbf{K}^{\prime}$ be an arbitrary closed $n^{\prime}$-braid representative. Then there is a finite sequence of closed braid representatives of $\boldsymbol{K}$ :

$$
\mathbf{K}=\mathbf{K}_{0} \rightarrow \mathbf{K}_{1} \rightarrow \mathbf{K}_{2} \rightarrow \cdots \rightarrow \mathbf{K}_{m}=\mathbf{K}^{\prime}
$$

Received by the editors December 10, 1988 and, in revised form, November 15, 1989.

1980 Mathematics Subject Classification (1985 Revision). Primary 57M25; Secondary 20F36.

Key words and phrases. Knot, link, closed braid, Markov equivalence, stabilization.

Early versions of this paper were entitled "Closed braid representatives of the unlink".

The partial support of the National Science Foundation under Grants \#85-03758 and DMS88-05672 is gratefully acknowledged by the first author. Part of this work was done during her sabbatical leave. She gratefully acknowledges the support of the University of Paris VII and the Institute for Advanced Study during that period. 


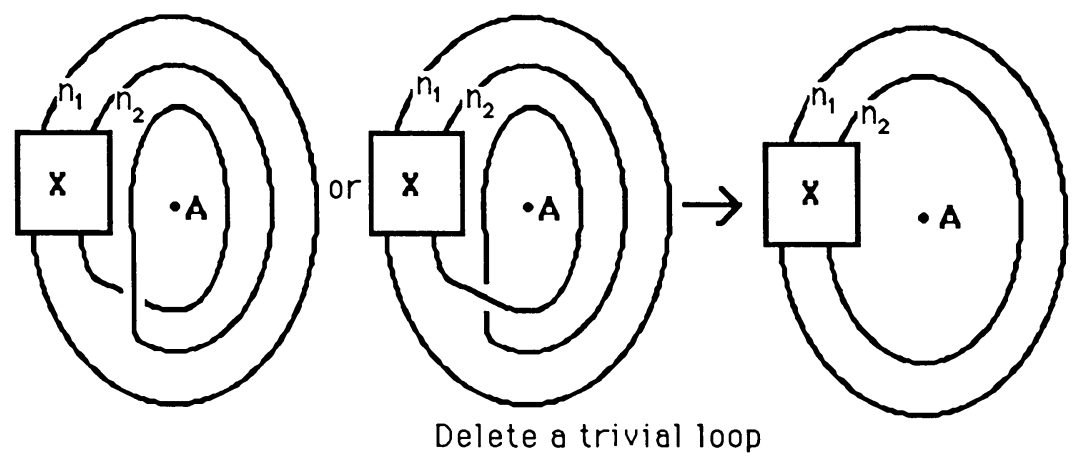

FIGURE 1

such that each $\mathbf{K}_{i+1}$ is obtained from $\mathbf{K}_{i}$ by one of the following moves:

(Mi) Addition or deletion of a trivial loop (see Figure 1).

(Mii) Isotopy in the complement of the axis (or, equivalently, conjugation in the braid group).

In the chain of closed braids which take $\mathbf{K}$ to $\mathbf{K}^{\prime}$ in Markov's theorem the braid index will in general go up to values larger than $n$, i.e. "stabilization" will occur. One may show without too much difficulty (although we shall not do so) that the braid indices $n_{i}$ of the braids $\mathbf{K}_{i}$ in the chain may be assumed to satisfy: $n_{1} \leq n_{2} \leq \cdots \leq n_{q} \geq n_{q+1} \geq n_{q+2} \geq \cdots \geq n_{m}$. The number $q$, however, seems to be elusive and in fact it has not even been well understood what is being accomplished by stabilization.

In the manuscript $[\mathrm{Mo}, 2]$ Hugh Morton gave an example of a 4-braid representative of the unknot which cannot be simplified to the trivial 1-braid representative without first increasing the string index to 5 . His example is of central importance in any attempt to understand all of the braid representatives of a link. The first reason is that it shows that stabilization is an essential part of Markov's theorem. The second reason is that, while Morton's example is special, any braid representative of any link may be changed to a new braid representative of the same link by connect-summing with a braid representative of the unknot, so that the phenomenon exhibited by his example is pervasive. A similar example for the 2-component unlink was given by Rudolph on p. 27 of $[R]$.

The work in this paper began with attempts to understand the role of stabilization in Morton's example. Ultimately, we were led to conjecture and prove a special version of Markov's theorem which avoids stabilization, in the case when $\boldsymbol{K}$ is the unlink of $r$ components. To state our results we introduce in Figure 2 the concept of an exchange move. Assume that the braid axis $\mathbf{A}$ is the $z$ axis, and that the arc which is labeled $n_{3}$ lies in the $x-y$ plane. Up to isotopy of $S^{3}$, an exchange is defined to be an isotopy of $\mathbf{K}$ which moves the arc which is labeled $n_{2}$ from a position which is a little bit above (or below) the $x-y$ plane to a position which is a little bit below (or above) the $x-y$ plane, keeping the rest of $\mathbf{K}$ invariant. The labels on the strands mean that a single strand which is labeled " $n_{i}$ " is to be replaced by $n_{i}$ parallel strands. We allow any type of braiding on the $n_{1}+n_{2}$ (respectively $n_{1}+n_{3}$ ) strands in the boxes which are labeled $X$ (respectively $Y$ and $Z$ ). An exchange move takes 


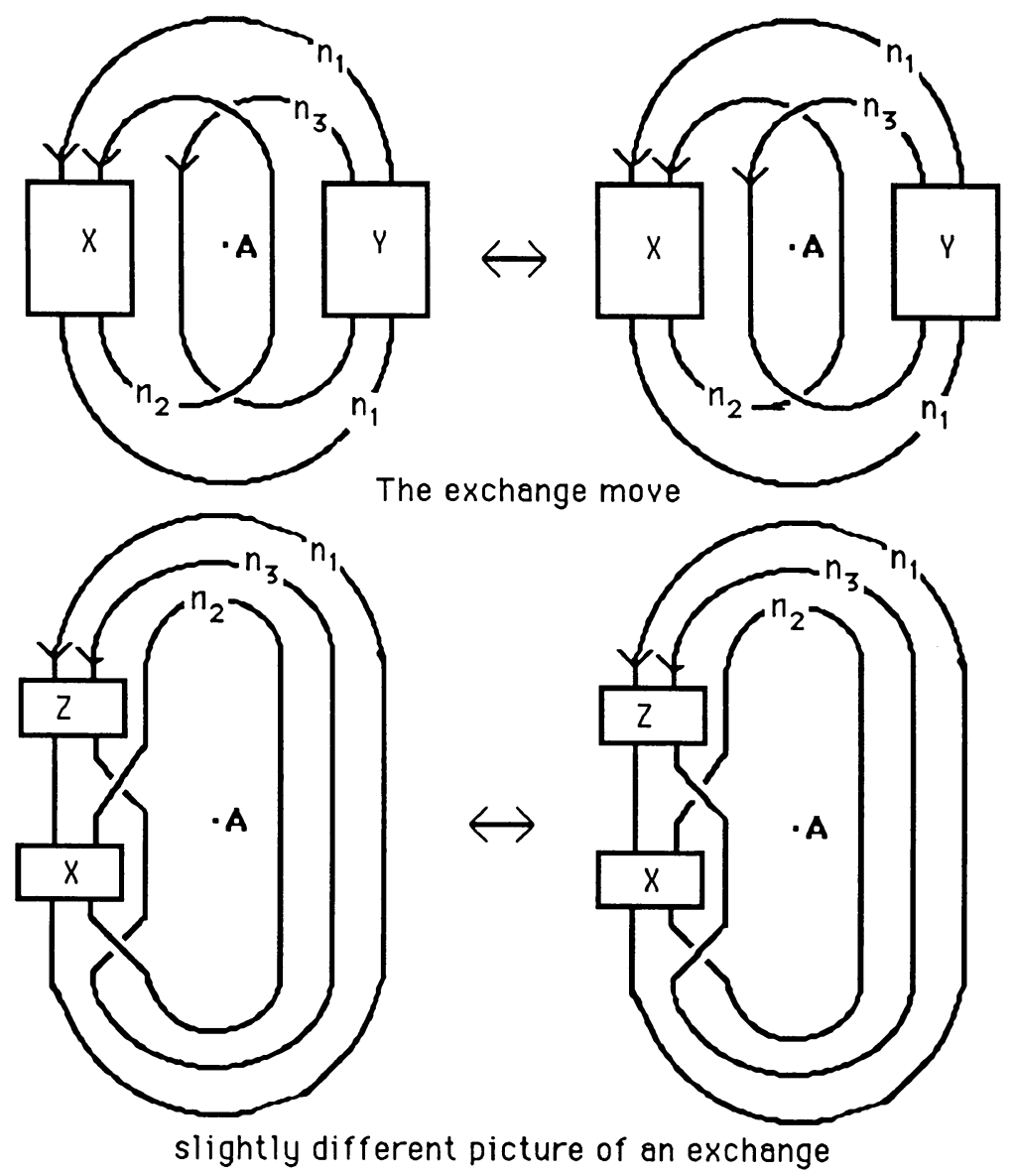

FIGURE 2

$n$-braids to $n$-braids, but need not preserve conjugacy class because the isotopy of $\mathbf{K}$ in $S^{3}$ is in general not realizable in the complement of the axis $\mathbf{A}$.

We remark that exchange moves are only applicable when a link admits a rather special type of braid representation. Figure 3 shows the projection of Figure 2, deformed so that the strands which are labeled $n_{2}$ and $n_{2}$ are pulled apart. There is then a 2-sphere $\mathbf{S}(C)$ which intersects $\mathbf{K}$ in two generalized points (or $n_{2}+n_{3}$ points) and is pierced twice by the axis $\mathbf{A}$. In the special case when $n_{2}=n_{3}=1$ the link $\mathbf{K}$ is composite and $\mathbf{S}(C)$ exhibits $\mathbf{K}$ as a "composite closed braid", so we say that any closed braid which admits an exchange is (up to conjugacy) a $G$-composite closed braid.

In $\S 2$ we will define a complexity function, with domain the collection of all closed braid representatives of the $r$-component unlink. Our main result is

Theorem 1. Let $\mathbf{K}$ be an arbitrary closed $n$-braid representative of the unlink of $r$ components, $r \geq 1$. Let $\mathbf{U}_{r}$ be the standard closed $r$-braid representative. Then there is a finite sequence of closed-braid representatives of the unlink:

$$
\mathbf{K}=\mathbf{K}_{0} \rightarrow \mathbf{K}_{1} \rightarrow \mathbf{K}_{2} \rightarrow \cdots \rightarrow \mathbf{K}_{m}=\mathbf{U}_{r}
$$

such that each $\mathbf{K}_{i+1}$ is obtained from $\mathbf{K}_{i}$ by one of the following moves: 


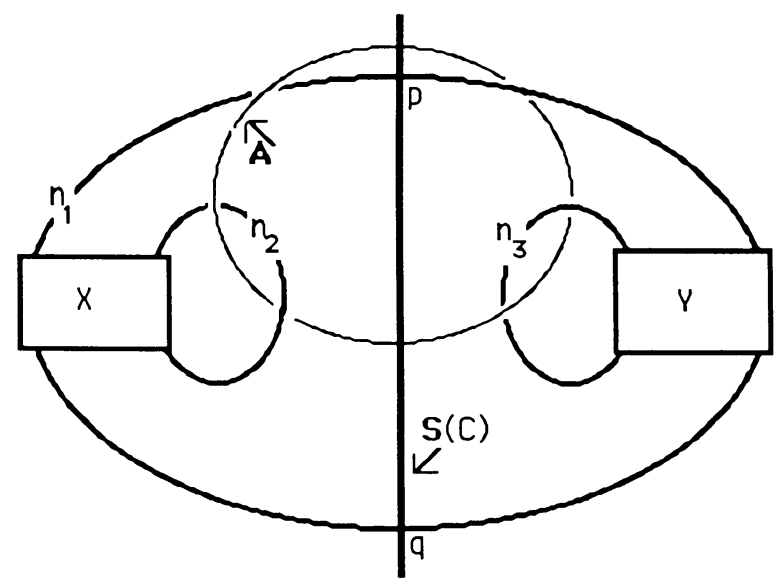

Figure 3

(i) A complexity-reducing exchange move.

(ii) A complexity-reducing deletion of a trivial loop.

(iii) A complexity-preserving isotopy in the complement of the axis (or, equivalently, conjugation in the braid group).

There are two challenging open problems suggested by Theorem 1 . The first is to estimate the complexity of an algorithm for recognizing the unlink, using the ideas introduced in the proof of Theorem 1. A second problem, in principal more difficult, is to find an explicit algorithm.

We can define the exchange move in terms of its effect on words in the generators of the braid group. See Figure 2 again. Let $1, \ldots, n$ denote the standard elementary braids which generate the $(n+1)$-strand braid group, and let $-i$ denote the inverse of $i$. If $n_{2}=n_{3}=1$, an exchange move is applicable if (and only if), up to conjugacy, the cyclic braid factorizes as $(Z)(n)(X)(-n)$, where $Z$ and $X$ do not involve the elementary braids $\pm n$. After the exchange the braid will factorize as $(Z)(-n)(X)(n)$. The general case, where the weights are not 1 , is easily worked out from this.

An example is in order. Using the notation just given, Morton's example from [Mo,2] is the cyclic braid $\mathbf{M}=-3-32-32111-21-2$. Replace $\mathbf{M}$ by its conjugate $\mathbf{M}^{\prime}=321 \mathbf{M}-1-2-3$ which is isotopic to the 4-braid $-2-21-23222-12-3$. See Figure 4 . We invite the reader to verify that $\boldsymbol{M}^{\prime}$ represents the unknot. By the argument given in Morton's paper, there is no way to modify $\mathbf{M}^{\prime}$ in the complement of the axis $\mathbf{A}$ to produce a trivial loop. Now, the cyclic braid $\mathbf{M}^{\prime}$ factorizes as $(Z)(3)(X)(-3)$, where $Z$ and $X$ depend only on the elementary braids $\pm 1, \pm 2$. This braid admits an exchange move which changes it to $(Z)(-3)(X)(3)$. A slightly different factorization of the cyclic braid word $(Z)(-3)(X)(3)$ exhibits it as $(C)(1)(D)(-1)$, where $C$ and $D$ depend only on $\pm 2, \pm 3$. Such a braid also admits an exchange, since by interchanging the origin and the point at infinity we switch the braid generators 1 and 3 , while leaving 2 fixed. The four crossings in $\mathbf{M}^{\prime}$ which are to be switched are indicated in Figure 4, the first pair enclosed in dotted circles and the second pair in dotted boxes. After this second exchange move 


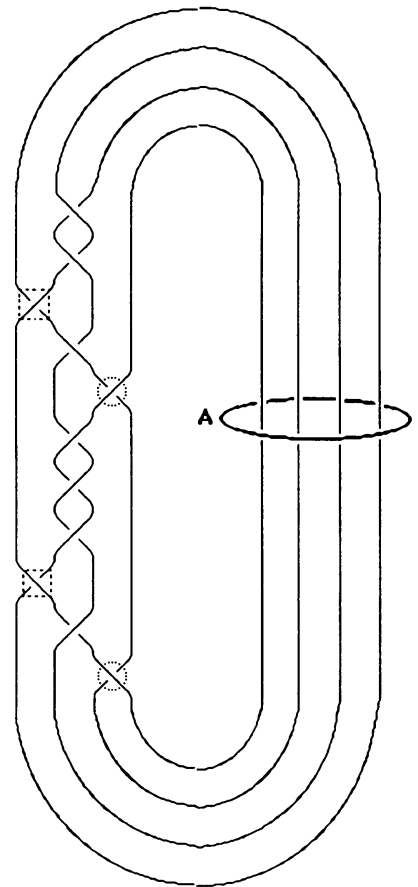

$M^{\prime}$

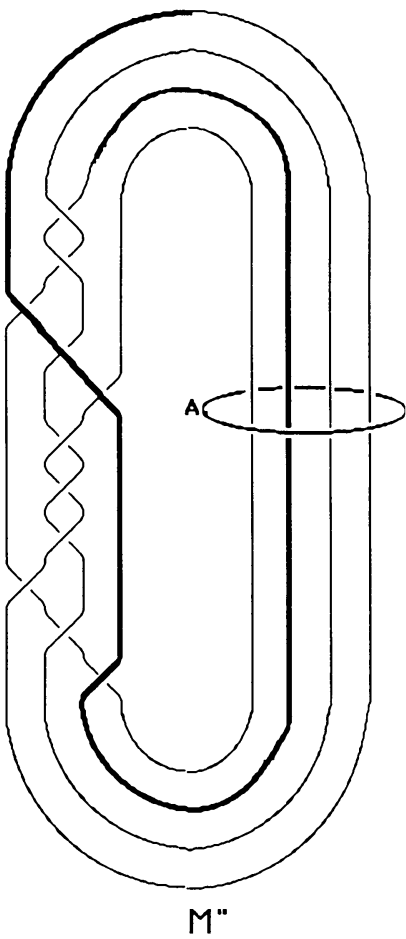

$M^{\prime \prime}$

FIGURE 4

we obtain $\mathbf{M}^{\prime \prime}=(C)(-1)(D)(1)=-2-2-1-2-3222123$, also depicted in Figure 4. It is easy to see that the portion of $\mathbf{M}^{\prime \prime}$ which is drawn in thickly can be modified by an isotopy in the complement of the axis to a trivial loop. It is known (see [Bi] or [Be] or $[\mathrm{Mo}, 1]$ ) that all closed 3-braid representatives of $\mathbf{U}_{r}$ are standard. This 4-braid example is the generic example which creates complications in closed braid representatives of the unlink.

Our Theorem 1 is a special case of "Markov's theorem without stabilization", and using it we can say something about the stabilization process. Let $\boldsymbol{K}$ be an arbitrary link, and let $n_{0}$ be the braid index of $\boldsymbol{K}$, i.e. the smallest integer $n$ such that $\boldsymbol{K}$ can be represented by an $n$-braid. (For the unlink, it is 1.) Choose $n \geq n_{0}$. A chain $C\left(\boldsymbol{K}, n, n_{0}\right)$ is a sequence of closed braid representatives of $K$, where the first member of the sequence is an $n$-braid and the last member is an $n_{0}$-braid and adjacent members are related by Markov's moves (Mi) and (Mii). Let $n^{\prime} \geq n$ be the maximum braid index occurring in the braids in $C\left(\boldsymbol{K}, n, n_{0}\right)$. Define the stabilization index $s(\boldsymbol{K}, n)$ to be the smallest value of the difference $\left(n^{\prime}-n\right)$, for all chains $C\left(K, n, n_{0}\right)$.

Corollary 2. The stabilization index $s\left(U_{r}, n\right)$ of the r-component unlink is one, for every $n$.

Caution. We do not give a bound on how many times it may be necessary to stabilize during a chain, and suspect that no such bound exists. We can construct examples of braid representatives of the unlink which seem to require arbitrarily many stabilizations (but each time only increasing the braid index by 1 , conjugating, and then decreasing it by 1 ) before they can be reduced to the standard 1-braid representative. 
Here is the plan of this paper. In $\S 2$ we standardize the geometry and set up our basic machinery. We will start with an arbitrary $n$-braid representative $\mathbf{K}$ of an $r$-component link, with braid axis $\mathbf{A}$. We choose a family $\mathbf{D}$ of $r$ disjoint embedded surfaces in 3-space with boundary $\mathbf{K}$. Experts will recognize the family D to be essentially a "Markov surface", as defined in [Be]. We begin to study the foliation of $\mathbf{D}$ which is induced by the fibration $\mathbf{H}$ of the open solid torus $S^{3}-\mathbf{A}$ by meridian discs. The leaves in this foliation are the components of $\mathbf{D} \cap \mathbf{H}_{t}$, as $t$ ranges over the interval $[0,2 \pi]$. After standardizing $\mathbf{D}$ and $\mathbf{H}$ we will be able to define a complexity function for the pair $(\mathbf{D}, \mathbf{H})$.

In $\S 3$ we prepare for the proof of Theorem 1 by showing that the foliation of $\mathbf{D}$ determines a foliated cell decomposition which we call a "tiling". The vertices of our tilings are the points where the axis $\mathbf{A}$ pierces $\mathbf{D}$, the edges are particular nonsingular leaves in the foliation, and the faces each contain one singularity. We define the notions of the type, sign, and valence of a vertex in the tiling. These concepts record information about the edges and the tiles which meet at that vertex. We show that combinatorial data in the tiling can be used to detect when our braid $\mathbf{K}=\partial \mathbf{D}$ has a trivial loop or admits an exchange.

In $\S 4$ we study the combinatorics of the tiling in greater detail. We prove several combinatorial lemmas which will be used to detect when we are in the situations of Lemmas 4,5 , and 6 . The ideas which are developed in $\S 2-4$ are used in $\S 5$ to prove Theorem 1 and Corollary 2 .

This study is part of a larger one in which the authors have been studying the closed braid representatives of links. Other papers in the series are [B-M,I; B-M,II; B-M,III; B-M,IV; B-M,VI]. This paper is self-contained, except that the proof of Lemma 6 is referred to the same result in [B-M,IV].

\section{BASIC MACHINERY}

We begin with an arbitrary $n$-braid representative $\mathbf{K}$ of the $r$-component unlink $\boldsymbol{U}_{r}, r \geq 1$, in oriented $S^{3}$. The braid axis is $\mathbf{A}$. We orient $\mathbf{A}$ so that $\mathbf{K}$ is oriented positively about $\mathbf{A}$, using the right-hand rule. Most of the time we will think of $\mathbf{A}$ as the $z$-axis in $R^{3}$.

Since $\mathbf{A}$ is unkotted, its complement $S^{3}-\mathbf{A}$ is an open solid torus, which can be fibered by meridian discs. It will be convenient to replace $S^{3}$ by $R^{3}$ and to think of the fibration as being by half-planes $\left\{\mathbf{H}_{t} ; t \in[0,2 \pi]\right\}$ of constant polar angle $t$ through the $z$-axis. Note that $\mathbf{K}$ intersects each half-plane $\mathbf{H}_{t}$ exactly $n$ times, transversally, and always from the negative to the positive side of $\mathbf{H}_{t}$, because $\mathbf{K}$ is a closed $n$-braid with axis $\mathbf{A}$.

Our link $\mathbf{K}$ is the boundary of a family of $q \leq r$ disjointly embedded discs $\mathbf{D}=\left\{\mathbf{D}_{1}, \ldots, \mathbf{D}_{q}\right\}$ in $S^{3}$. The disks $\mathbf{D}$ are assumed to be oriented so that the positive normal bundle to each component of $\mathbf{D}$ has the orientation induced by that on $\mathbf{K}=\partial \mathbf{D}$. By standard general position arguments we may assume:

(i) The intersections of $\mathbf{A}$ with $\mathbf{D}$ are finite in number and transverse.

(ii) There is a solid torus neighborhood $\mathbf{N}(\mathbf{A})$ of $\mathbf{A}$ in $S^{3}-\mathbf{K}$ such that each component of $\mathbf{D} \cap \mathbf{N}(\mathbf{A})$ is a disc. The foliation in each component of $\mathbf{D} \cap \mathbf{N}(A)$ is the standard radial foliation.

(iii) All but finitely many of the fibers $\mathbf{H}_{t}$ of $\mathbf{H}$ meet $\mathbf{D}$ transversally, and those which do not (the singular fibers) are each tangent to $\mathbf{D}$ at exactly one point in the interior of both $\mathrm{D}$ and $\mathbf{H}_{t}$. 

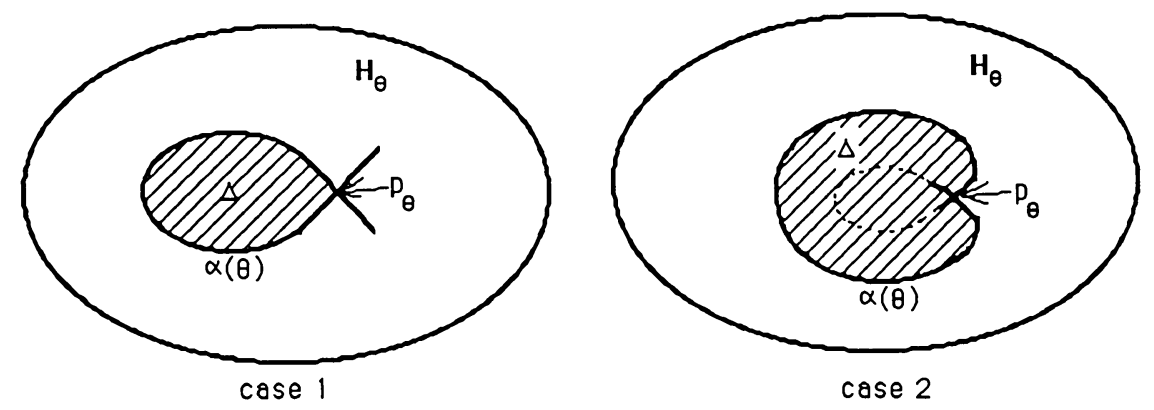

FIGURE 5

(iv) The tangencies in (iii) are local maxima or minima or saddle points.

The complexity $c(\mathbf{D}, \mathbf{H})$ of the pair $(\mathbf{D}, \mathbf{H})$ is defined to be:

$$
c(\mathbf{D}, \mathbf{H})=(|\mathbf{A} \cap \mathbf{D}|,|\mathbf{H} \cdot \mathbf{D}|),
$$

where $|\mathbf{A} \cap \mathbf{D}|$ is the number of points in $\mathbf{A} \cap \mathbf{D}$ and $|\mathbf{H} \cdot \mathbf{D}|$ is the number of tangencies between fibers of $\mathbf{H}$ and our surface $\mathbf{D}$. We assign the standard lexicographic ordering to this complexity function. The pairs $(\mathbf{D}, \mathbf{H}),\left(\mathbf{D}^{\prime}, \mathbf{H}\right)$ will be said to be equivalent if there is an isotopy of $\left(\mathbf{D} \cap \mathbf{H}_{t}, \mathbf{D} \cap \partial \mathbf{H}_{t}\right)$ to $\left(\mathbf{D}^{\prime} \cap \mathbf{H}_{t}, \mathbf{D}^{\prime} \cap \partial \mathbf{H}_{t}\right)$, for all $t \in[0,2 \pi]$. By our definition of equivalence, every representative of an equivalence class has the same complexity. Thus $c(\mathbf{D}, \mathbf{H})$ is well defined on equivalence classes.

By our assumptions (i)-(iv) above each component of $\mathbf{D} \cap \mathbf{H}_{t}$, where $\mathbf{H}_{t}$ is nonsingular, is an arc or a simple closed curve.

Lemma 1 [Be]. Assume that (D, H) satisfies (i)-(iv) and has simple closed curves. Then there exists $\left(\mathbf{D}^{\prime}, \mathbf{H}\right)$, with $\partial \mathbf{D}^{\prime}=\partial \mathbf{D}$, such that $\left(\mathbf{D}^{\prime}, \mathbf{H}\right)$ also satisfies (i)-(iv) and has no simple closed curves. Moreover, $c\left(\mathbf{D}^{\prime}, \mathbf{H}\right) \leq c(\mathbf{D}, \mathbf{H})$.

Proof of Lemma 1. The proof is essentially given on pp. 106-107 of [Be]. Since it is basic to our work, does not take long to explain, and appears to be unfamiliar to most of our readers we repeat Bennequin's argument here.

Suppose there is a scc $\alpha(t)$ in $\mathbf{D} \cap \mathbf{H}_{t}$ for some nonsingular $\mathbf{H}_{t}$. Since each component of $\mathbf{D}$ is pierced by the braid axis and since the foliation is radial near the axis, all of $\mathbf{D}$ cannot be foliated by simple closed curves. Therefore if we follow $\alpha(t)$ as it evolves in the fibration, we must arrive in one direction at an $\alpha(\theta)$ which contains a singular point $p_{\theta}$ of the foliation. The point $p_{\theta}$ is either a center or a saddle point, but if it is a center we can reverse the direction of the flow and find a saddle. So, we may assume it is a saddle. By property (iii) the singular point $p_{\theta}$ is the only singularity on the fiber $\mathbf{H}_{\theta}$, so the singularity is necessarily a homoclinic point (Figure 5). There are two possibilities, illustrated in Figure 5, but if the second occurs then the first occurs for some other choice of $\alpha(t)$, so we may assume we are in the situation of case 1 . Thus $\alpha(\theta)$ bounds a disc $\Delta$ in $\mathbf{H}_{\theta}$ and another disc $\Delta^{\prime}$ in $\mathbf{D}$. Their union $\Delta \cup \Delta^{\prime}$ is a 2-sphere $S$ in $S^{3}$, and the algebraic intersection number of $\mathbf{K}$ with $S$ is necessarily zero.

Since $\mathbf{K}$ does not intersect the interior of $\mathbf{D}$, it also does not intersect $\Delta^{\prime}$. From this it follows that $\Delta$ also cannot be punctured by $\mathbf{K}$. For, the algebraic and geometric intersection numbers of $\mathbf{K}$ with $\Delta$ coincide, because $\Delta$ is a subdisc of $\mathbf{H}_{\theta}$ and $\mathbf{K}$ is a closed braid with axis $\partial \mathbf{H}_{\theta}$. Therefore the geometric 


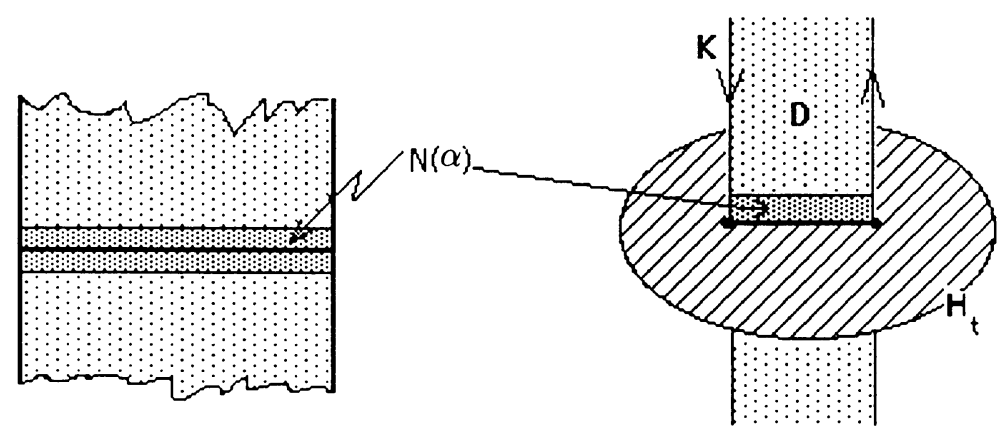

(a)

(b)

Figure 6

intersection number of $\Delta$ with $\mathbf{K}$ is equal to the algebraic intersection number of $\mathbf{K}$ with $S$, i.e. 0 , and from this it follows that $\mathbf{K}$ does not pierce $\Delta$.

If the interior of $\Delta$ has empty intersection with $\mathbf{D}$, we surger $\mathbf{D}$ along $\Delta$ and discard the closed component. If the interior of $\Delta$ meets $\mathbf{D}$, we find an innermost subdisc $\delta$ of $\Delta$ whose boundary is a component of $\mathbf{D} \cap \mathbf{H}_{\theta}$ and surger $\mathbf{D}$ along $\delta$. Ultimately, we will arrive at a new disc $\mathbf{D}^{\prime}$ which has the property that the induced foliation has no simple closed curves, and $c\left(\mathbf{D}^{\prime}, \mathbf{H}\right) \leq$ $c(\mathbf{D}, \mathbf{H})$.

Lemma 2. An arc in $\mathbf{H}_{t} \cap \mathbf{D}$, where $\mathbf{H}_{t}$ is nonsingular, cannot have both of its endpoints on $\mathbf{K}$.

Proof of Lemma 2. Let $\alpha \in \mathbf{H}_{t} \cap \mathbf{D}$ be an arc which has both of its endpoints on $\mathbf{K}$, and let $\mathbf{N}(\alpha)$ be a neighborhood of $\alpha$ on $\mathbf{D}$. See Figure 6. Then $\mathbf{K} \cap \mathbf{N}(\alpha)$ has two components, $\kappa$ and $\kappa^{\prime}$, which have opposite orientations as subarcs in the boundary of the oriented surface $\mathbf{N}(\alpha)$. However, $\mathbf{K}$ is a closed $n$-braid, hence it meets each $\mathbf{H}_{t}$ in $n$ coherently oriented transverse intersections. Since $\alpha$ lies in both $\mathbf{D}$ and $\mathbf{H}_{t}$, and since $\mathbf{N}(\alpha)$ intersects $\mathbf{H}_{t}$ transversally, this is impossible.

In view of Lemma 1 we may replace condition (iv) by the stronger condition.

(v) Each component of $\mathbf{H}_{t} \cap \mathbf{D}$, where $\mathbf{H}_{t}$ is nonsingular, is an arc. Each tangency of a singular fiber with $\mathbf{D}$ is a saddle-point tangency.

The arcs in $\mathbf{H}_{t} \cap \mathbf{D}$, where $\mathbf{H}_{t}$ is nonsingular, are of two types. The first type has both endpoints on $\mathbf{A}=\partial \mathbf{H}_{t}$ (call it an a-arc). The second type has one of its endpoints on $\mathbf{A}=\partial \mathbf{H}_{t}$ and the other on $\mathbf{K}=\partial \mathbf{D}$ (call it a b-arc). A b-arc, $b_{i}$, in a disc fiber $\mathbf{H}_{t}$ is said to be essential if, when $\mathbf{H}_{t}$ is split along $b_{i}$, both components contain points of $\mathbf{H}_{t} \cap \mathbf{K}$. For example, the arc $b_{1}$ the two top sketches in in Figure 7 is essential, while $b_{2}$ and $b_{3}$ are not. Note that the link itself is the only possible obstruction to the removal of a $\mathbf{b}$-arc, therefore any time we have an essential $\mathbf{b}$-arc we know that $\mathbf{K}$ must loop around the axis as in the bottom sketch in Figure 7, in between the two pierce-points.

Lemma 3. Given any pair $(\mathbf{D}, \mathbf{H})$, there exists a pair $\left(\mathbf{D}^{\prime}, \mathbf{H}\right)$ with $c\left(\mathbf{D}^{\prime}, \mathbf{H}\right) \leq$ $c(\mathbf{D}, \mathbf{H})$ such that $\left(\mathbf{D}^{\prime}, \mathbf{H}\right)$ has only essential $\mathbf{b}-$ arcs.

Proof of Lemma 3. If $\mathbf{D}$ has an inessential $\mathbf{b}$-arc, that arc will cobound with a segment of the axis $\mathbf{A}$ a disc $\Delta$ in some $\mathbf{H}_{t}$. See Figure 7. We can then push 

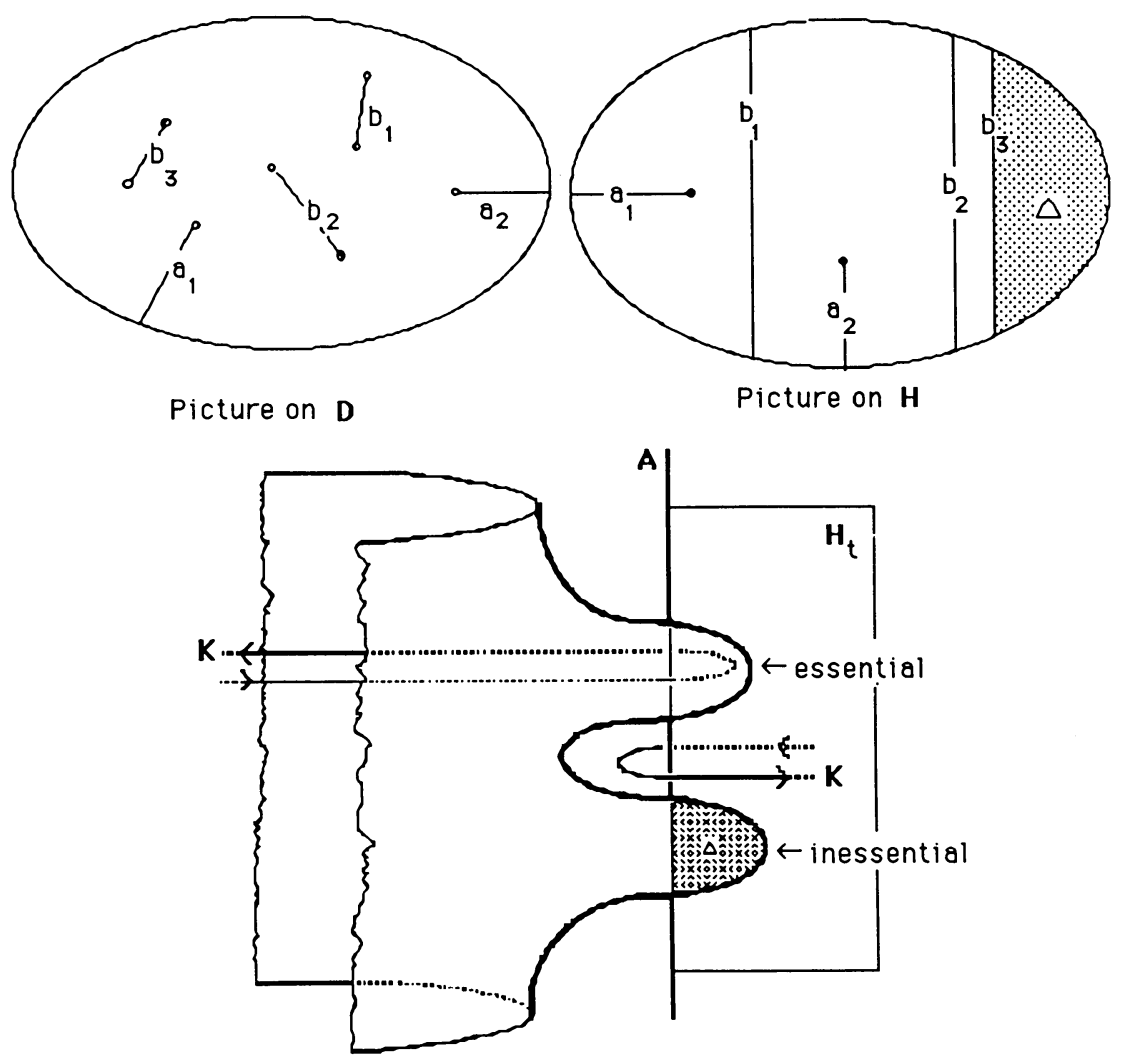

Picture in 3-space

FIGURE 7

D in along a neighborhood of $\Delta$ in $S^{3}$ to remove two points of intersection of $\mathbf{D}$ with the axis $\mathbf{A}$. This process may introduce simple closed curves into the foliation of $\mathbf{D}$, however if so they can be removed with the aid of Lemma 1. The complexity will be reduced even if simple closed curves are introduced because the removal of inessential b-arcs reduces $|\mathbf{A} \cap \mathbf{D}|$.

A pair (D, H) is said to be a good pair if $\mathbf{D}$ satisfies properties (i)-(iii) and (v), and if all b-arcs are essential. By what we have just shown we may assume we have a good pair.

\section{TILINGS}

We will show in this section that the foliation of $\mathbf{D}$ determines a type of foliated cell decomposition of $\mathbf{D}$ which we call a tiling. In Lemma 4 we show that the tiling enables us to recognize when our closed braid $\mathbf{K}$ has a trivial loop. In Lemmas 5 and 6 we show that the tiling can be used to recognize when $\mathbf{K}$ admits an exchange.

We begin our work by investigating the singularities in the foliation of $\mathbf{D}$. Since $(\mathbf{D}, \mathbf{H})$ is a good pair the components of $\mathbf{D} \cap \mathbf{H}_{t}$, for each nonsingular $\mathbf{H}_{t}$, will be a union of a-arcs and essential $\mathbf{b}$-arcs. Since we have two types of arcs, a-arcs and $\mathbf{b}$-arcs, there are at most three types of singularities: 

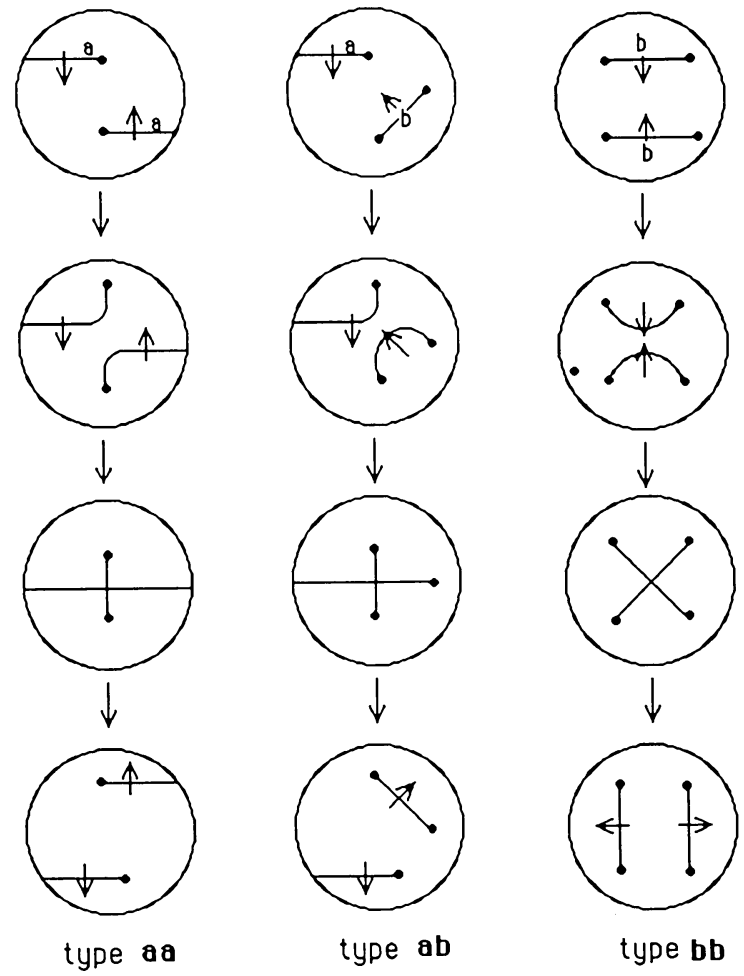

FIGURE 8. Singularities in the foliation

type aa: an a-arc surgered with an a-arc,

type $a b$ : an a-arc surgered with a $\mathbf{b}$-arc,

type $b b$ : a b-arc surgered with a $\mathbf{b}$-arc.

We assume that the singular points of the foliation occur at the interiors of the arcs which are involved. The left column in Figure 8 shows a sequence of leaves in $\mathbf{D}$ before and after a type aa singularity, where since $\mathbf{D}$ is an oriented surface we can assume we are looking at its positive side $\mathbf{D}^{+}$. Figure 9 shows the induced foliation on $\mathbf{D}^{+}$. The arrows indicate the direction of increasing $t$. Notice that the foliation on $\mathbf{D}$ forms a foliated 2-cell whose vertices are the two pierce-points, whose edges are four (nonsingular) a-arcs with two $p$-endpoints, and whose interior contains a single type aa singularity. We will call such a 2-cell an aa-tile. Its edges can be any convenient choice of nonsingular a-arcs.

The middle column in Figure 8 shows a sequence of leaves in $\mathbf{D}^{+}$just before and after a type ab singularity. Figure 9 shows the induced foliation on $\mathbf{D}^{+}$. Again, notice that the foliation on $\mathbf{D}^{+}$forms a tile whose sides are two a-arcs and two $b$-arcs with three $p$-endpoints, and whose interior contains a type ab singularity. We thus have an ab-tile.

Finally, the right column in Figure 8 shows a sequence of leaves of $\mathbf{D}^{+}$before and after a type bb singularity, and Figure 9 shows the induced foliation on $\mathbf{D}^{+}$. The $b b$-tile formed by the foliation on $\mathbf{D}^{+}$has four $\mathbf{b}$-arcs as its sides with four $p$-endpoints, and its interior contains a type bb singularity. 

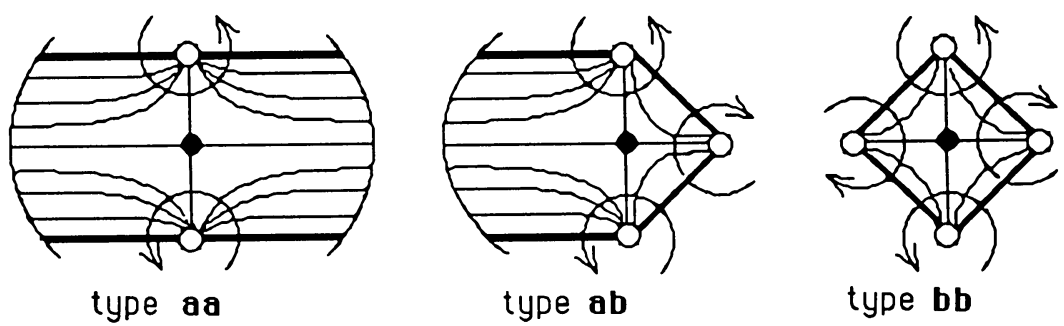

Figure 9. The three tile-types on D
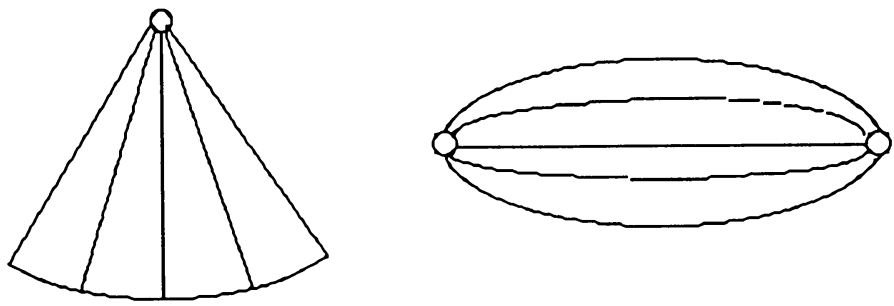

FIGURE 10. Foliated regions in the complement of the singular arcs

A component $\mathbf{D}_{i}$ of $\mathbf{D}$ will be said to be trivially tiled if $\mathbf{K}_{i}=\partial \mathbf{D}_{i}$ is a 1-braid, so that $\mathbf{D}_{i}$ is pierced once by the axis $\mathbf{A}$ and has the standard radial foliation, which has no singularities. A tiling of a component of $\mathbf{D}_{i}$ of $\mathbf{D}$ is either the trivial tiling, or else it is a subdivision of $\mathbf{D}_{i}$ into a union of tiles of type $a a, a b$, and $b b$. Here is a way to see that tilings exist. Let $\mathbf{U}$ be the union of all of the singular leaves in the foliation of $\mathbf{D}$. Then each component of $\mathbf{D}-\mathbf{U}$ is a union of regions $\mathbf{R}_{1}, \ldots, \mathbf{R}_{q}$, each of which is foliated in one of the two ways shown in Figure 10. We choose a leaf from the foliation of each region $\mathbf{R}_{j}$, and declare it to be a tile edge. The union of all of the edges determines a tiling of $\mathbf{D}$. Note that this also shows that any two tilings necessarily agree up to the choice of the particular edges in the regions $\mathbf{R}_{1}, \ldots, \mathbf{R}_{q}$.

Our tiling gives a foliated cell decomposition of $\mathbf{D}$. The vertices are the points $p_{1}, \ldots, p_{n+2 \mu}$ where $\mathbf{A}$ pierces $\mathbf{D}$. (Remark: we will not need to record the vertices or edges which are on $\partial \mathbf{D}$.) The faces are foliated tiles of type aa, $\mathrm{ab}$, and $\mathrm{bb}$. Each face contains exactly one singularity of the foliation. The edges of the tiles are nonsingular leaves of the foliation. We regard two tilings as being equivalent if they differ only in the choice of the tile edges. Clearly equivalent tilings give equivalent cell decompositions, foliated in the same way. The valence of a vertex is the number of tile edges which meet at that vertex. The type symbol of a vertex is a cyclic sequence $\left(x_{1}, x_{2}, \ldots, x_{v}\right)$ where each $x_{i}$ is either $a$ or $b$ and the cyclic sequence describes the types of the tile edges which meet at that vertex.

The next lemma shows how combinatorial properties of the tiling can be used to detect the presence of a trivial loop.

Lemma 4. Let $\mathbf{D}_{i}$ be a component of $\mathbf{D}$ which admits a nontrivial tiling. Suppose that tiling contains a vertex of valence 1 . Then that vertex has type (a) and the braid $\mathbf{K}$ has a trivial loop. Removing the trivial loop preserves link type and reduces $c(\mathbf{D}, \mathbf{H})$. 


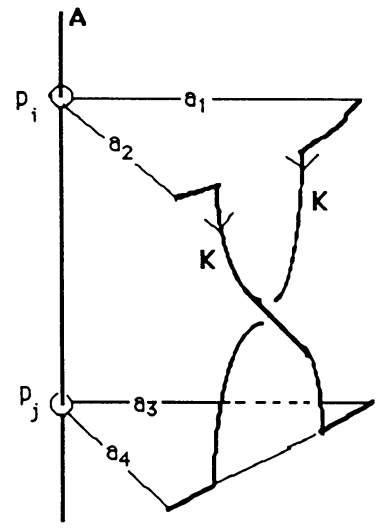

typical ao-tile embedded in $S^{3}$

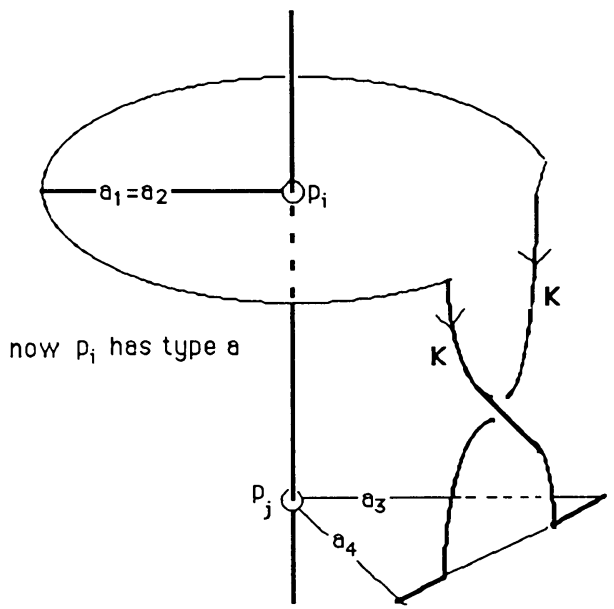

Figure 11

Proof of Lemma 4. By hypothesis the tiling of $\mathbf{D}_{i}$ contains a vertex of valence 1. A vertex of valence 1 is either type (a) or type (b), so assume first that it is type (b). Then there is a type bb (respectively ab) tile in the tiling of $\mathbf{D}_{i}$ which has two adjacent $b$-edges identified, and so has only 3 (resp. 2) distinct vertices instead of 4 (resp. 3). Now our tile contains exactly one singular point. This singularity occurs when two nonsingular leaves come together. This means that just before the singularity there are two components of $\mathbf{H}_{t} \cap \mathbf{D}_{i}$ emerging from a single point of $\mathbf{A} \cap \mathbf{D}_{i}$, which is impossible.

Now suppose that $\mathbf{D}_{i}$ has a vertex of type (a). Since the only tile which has a pair of adjacent a-edges meeting at a common vertex is an aa-tile, it follows that the vertex in question must belong to an aa-tile. Call it $X$. We study how $X$ is embedded in $S^{3}$. Assume that $\mathbf{A}$ and $\mathbf{H}$ are standard. From Figure 9 we know that the axis $\mathbf{A}$ pierces $X$ from the same side at the two pierce-points $p_{i}$ and $p_{j}$. From Figure 9 we also see that the foliation of $X$ has a unique separating leaf through the singular point. Note that if the vertices $p_{i}$ and $p_{j}$ are specified as particular points on $\mathbf{A}$ and if the singular point is specified as being on a particular fiber and having a particular sign, then the rest of the embedding of $X$ in $S^{3}$ will be completely determined by the leaves of the foliation, which are level sets for the embedding. The first picture in Figure 11 shows how this embedding must look when $p_{i}$ and $p_{j}$ both have valence $>1$. Our tile necessarily has an odd number of twists because the axis pierces the same side of $X$ at $p_{i}$ and $p_{j}$. There is only one twist because any additional twists would create additional tangencies with $\mathbf{H}$. The singular point occurs at the midpoint of the twist in the band. The second picture in Figure 11 shows how things will be modified if the vertex $p_{i}$ has valence 1 . Notice that in this case the foliation will be standard in the entire subdisc which contains $p_{i}$ and is cut off from $\mathbf{D}$ by the separating leaf, so there must be a trivial loop around A near $p_{i}$.

We can associate a sign to each tile, in the following way. Each tile contains exactly one singular point of the foliation. At the singular point the normals to D and to the singular fiber coincide as unoriented lines, however their orienta- 
tions may either agree or disagree. We define the sign of the tile to be positive if they agree, otherwise negative. The sign of a vertex in the tiling is the cyclic array of signs of the tiles which meet at that vertex.

The next two lemmas will be very important in our work. They tell us how to recognize from combinatorial properties of the tiling when our link admits a complexity-reducing exchange move.

Lemma 5. Assume that the tiling of some component $\mathbf{D}_{i}$ of $\mathbf{D}$ contains a vertex of type $(\mathrm{ab})$ and sign $(+,-)$. Then $\mathbf{K}=\partial \mathbf{D}$ admits an exchange. Moreover, after the exchange the complexity can be reduced by removing two of the points where the axis A pierces $\mathbf{D}$.

Proof of Lemma 5. Let $p_{0}$ be the given vertex of type $(\mathrm{ab})$ and sign $(+,-)$. Such a vertex occurs only when two type ab tiles are identified along corresponding $\mathbf{a}$ and $\mathbf{b}$ edges, as depicted in the top picture in Figure 12.

The vertex $p_{0}$ is joined to a second vertex $p_{1}$ by a type $\mathrm{b}$ tile edge, and $p_{0}$ is joined to $\partial \mathbf{D}$ by a type a tile edge. We know exactly how the foliation looks in our two tiles from our earlier work, i.e. from Figure 9. We also know that the foliation is standard in a neighborhood of $p_{1}$ (Figure 6). We may then find an arc $\alpha^{\prime}$ (Figure 12) which begins and ends on $\mathbf{K}$ and is everywhere transverse to the foliation, which cobounds with a subarc $\alpha$ of $\mathbf{K}$ a subdisc $\Sigma$
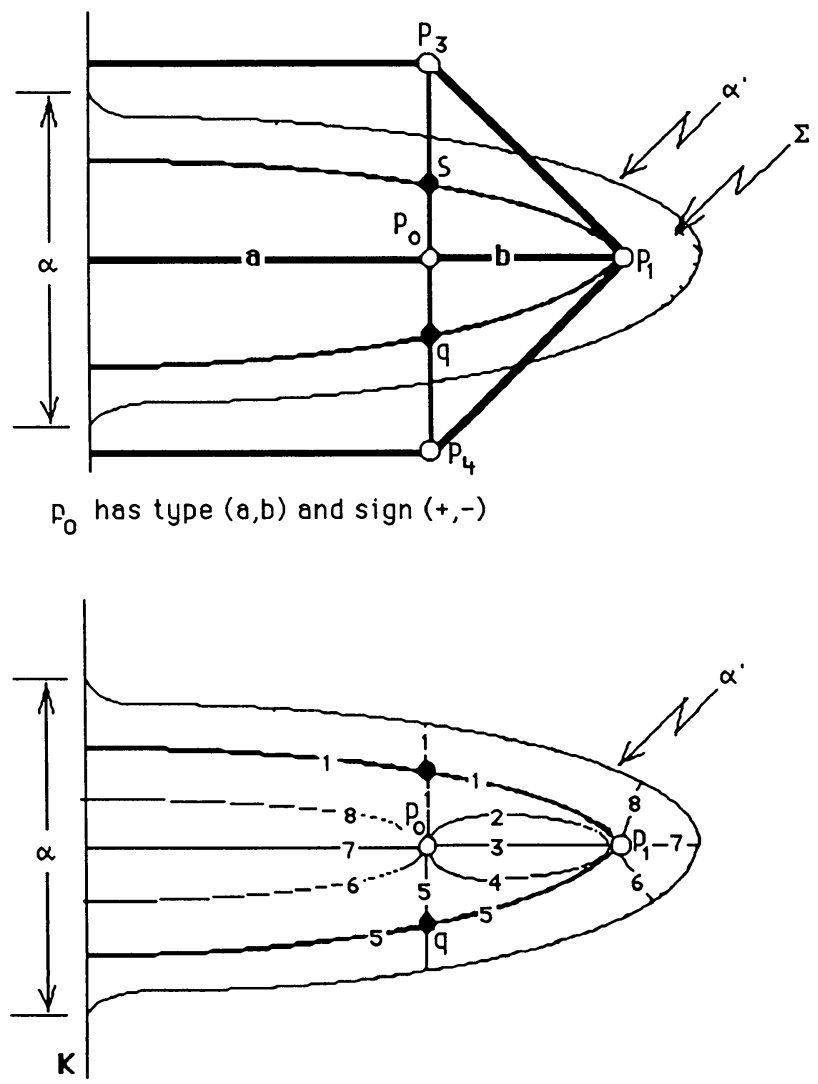

FIGURE 12 


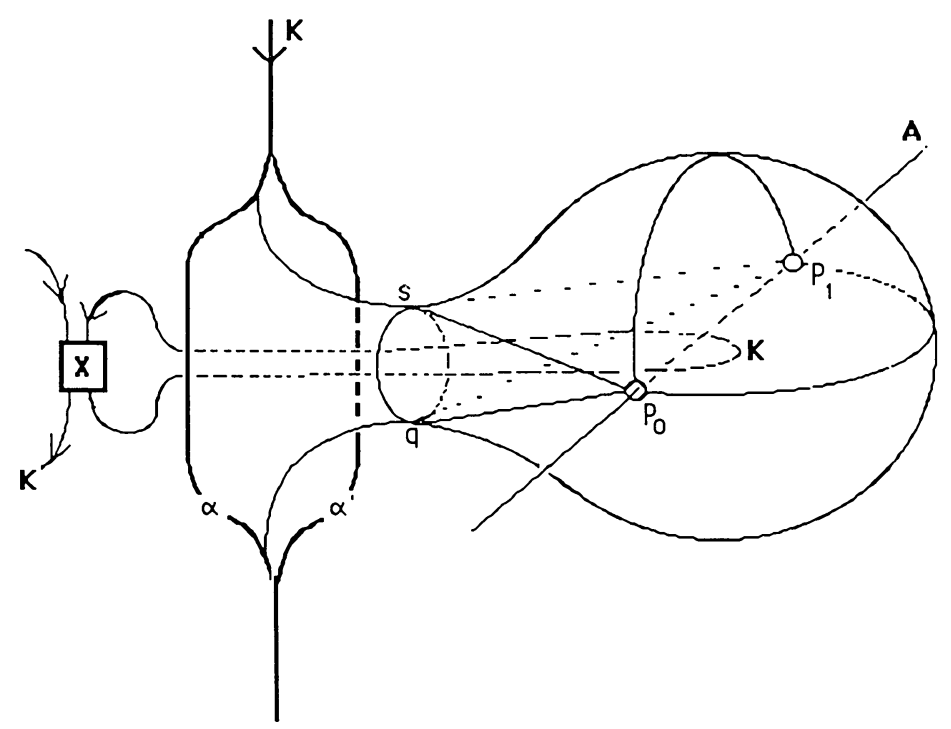

FIGURE 13. The disc $\Sigma \subset S^{3}$

of $\mathbf{D}$, where $\Sigma$ contains the two singular points $s$ and $q$. We can then push $\alpha$ across $\Sigma$ to $\alpha^{\prime}$, crossing the axis twice as we do so, to obtain a new embedding $\mathbf{K}^{\prime}=\mathbf{K}-\alpha+\alpha^{\prime}$ of $\mathbf{K}$ in $S^{3}$.

Clearly $\mathbf{K}$ also represents $\boldsymbol{U}_{r}$ because $\mathbf{K}^{\prime}$ is isotopic to $\mathbf{K}$ in $S^{3}$ (but not in $S^{3}-\mathbf{A}$ ). Since $\alpha$ was chosen to be everywhere transverse to the leaves of the foliation, it follows that $\mathbf{K}^{\prime}$ will be everywhere transverse to the disc fibers of $\mathbf{H}$, therefore $\mathbf{K}^{\prime}$ is a closed braid. Note that the sense of rotation of the flow about the pierce-point $p_{0}$ in opposite to that about $p_{1}$ by the conventions described in $\S 2$. From this it follows that the braid index of $\mathbf{K}^{\prime}$ is $n$, because the algebraic rotation number of the flow about the deleted pierce-points is zero, so the algebraic rotation number of $\mathbf{K}^{\prime}$ about $\mathbf{A}$ is the same as that of $\mathbf{K}$ about $\mathbf{A}$, namely $n$. Since $\mathbf{D}^{\prime}$ intersects the axis $\mathbf{A}$ two times less than $\mathbf{D}$, we have reduced the complexity. So, it only remains to show that $\mathbf{K}^{\prime}$ and $\mathbf{K}$ are related by an exchange move.

With that goal in mind we pass to a picture in 3-space. The bottom picture in Figure 12 shows $\Sigma$ again. We have sketched in leaves of the foliation around $p_{0}$ and $p_{1}$, and labeled them $1,2, \ldots, 8$ according to their polar angles $t_{1}, t_{2}, \ldots, t_{8}$. This figure will be helpful as we proceed. Also, consult Figure 13, as we begin to determine the embedding. The first step in embedding $\Sigma$ in 3-space is to choose points on $\mathbf{A}$ and declare them to be the pierce-points $p_{0}$ and $p_{1}$. Let $\delta_{0}$ and $\delta_{1}$ be little disc neighborhoods of $p_{0}$ and $p_{1}$ on $\Sigma$. By our earlier hypotheses, $\delta_{0}$ and $\delta_{1}$ will be transverse to $\mathbf{A}$ and radially foliated. The flow is in opposite directions about $p_{0}$ and $p_{1}$ (see Figure 9) so when we embed $\delta_{0}$ and $\delta_{1}$ in 3-space, they will be pierced from opposite sides by $\mathbf{A}$.

The second step is to choose two half-planes through $\mathbf{A}$ and declare them to be the fibers $\mathbf{H}_{s}$ and $\mathbf{H}_{q}$ which contain the singular points $s$ and $q$, and also to choose points on $\mathbf{H}_{s}$ and $\mathbf{H}_{q}$ and declare them to be $s$ and $q$. Now, the singular leaves go out from $s$ and $q$, in $\mathbf{H}_{s}$ and $\mathbf{H}_{q}$, like the spokes on a wheel, with an adjacent pair of singular leaves which begin at $s$ terminating 
at $p_{0}$ and $p_{1}$, and similarly at $q$. The leaves which go out from $s$ and also from $q$ will have their endpoints on $\alpha^{\prime}, p_{1}, p_{0}, \mathbf{K}$ respectively. However the positive normal to $\mathbf{D}$ agrees with that to $\mathbf{H}$ at, say, $s$ and disagrees at $q$, because the sign of $p_{0}$ is $(+,-)$, so the cyclic order of the spokes as seen on $\mathbf{H}_{s}$ will be opposite to that seen on $\mathbf{H}_{q}$.

Having embedded the singular leaves $\mathbf{S}$ in $\Sigma$, we now observe that $\Sigma-\mathbf{S}$ is transverse to every fiber. It is easy to extend the embedding to an embedding of the rest of $\Sigma$ because the leaves of the foliation are level sets for the embedding. The result is the picture in Figure 13.

All b-arcs in the foliation are essential. This means that the link itself must be an obstruction to their removal, and we have shown it to be so in Figure 13. We can push all braiding away from $\mathbf{A}$, into the box labeled $\mathbf{X}$. Projection parallel to $\mathbf{A}$ now clearly shows that when we push $\alpha$ across $\Sigma$ to $\alpha^{\prime}$, we are doing an exchange move, as defined in $\S 1$. This completes the proof of Lemma 5.

Lemma 6 (Lemma 4 of [B-M,IV]). Assume that the tiling of a component $\mathbf{D}_{i}$ of $\mathbf{D}$ contains a vertex of type (bb). Then $\mathbf{K}$ admits an exchange. Moreover, after the exchange the complexity can be reduced by removing two of the points where the axis pierces $\mathbf{D}$.

Proof of Lemma 6. See [B-M,IV]. The proof is very similar in flavor to the proof we have just given of Lemma 5.

\section{COMBINATORICS OF THE TILING}

In this section we will study detailed properties of the tiling. The reader should keep the statements of Lemmas 4, 5, and 6 firmly in mind in order to understand the goals in this section, which are to prove that in certain situations vertices of type $(a),(b b)$, or $(a b)$ with appropriate signs exist.

Lemma 7. Let $\mathbf{D}_{j}$ be a component of $\mathbf{D}$ which is nontrivially tiled. Assume that the tiling of $\mathbf{D}_{j}$ contains no vertex of valence 1 . Then the tiling of $\mathbf{D}_{j}$ always contains a vertex of type $(\mathrm{ab})$ or $(\mathrm{bb})$ or $(\mathrm{bbb})$.

Proof of Lemma 7. Let $V$ be the number of vertices, $E$ the number of edges, and $F$ the number of faces in the tiling of $\mathbf{D}_{j}$. (Recall that we do not count vertices or edges which are on $\partial \mathbf{D}$.) Let $\mathbf{F}_{j}$ be the 2-sphere which is obtained from $\mathbf{D}_{j}$ by collapsing the boundary of $\mathbf{D}_{j}$ to a point. Examining how our three tile types are modified by the collapsing map, we see that the tiling of $\mathbf{D}_{j}$ induces a cellular decomposition of $\mathbf{F}_{j}$, with $V+1$ zero-cells, $E$ one-cells, and $F$ two-cells, so that $V+1-E+F=2$. Examining how our three tile types were modified by the collapsing map, we see that every 2-cell in the tiling of $\mathbf{F}_{j}$ has four edges, also every 1-cell is adjacent to two 2-cells, so $2 F=E$. Thus $V$ and $E$ are related by

$$
2 V-E=2 .
$$

To get a sharper result, let $V(\alpha, \beta)$ denote the number of vertices in the tiling of $\mathbf{D}_{j}$ which have $\alpha$ a-arcs and $\beta$ b-arcs in their type symbols. Let $v$ be the valence of a vertex. Then $\alpha+\beta=v$, so that $V(\alpha, v-\alpha)$ denotes the number of vertices in the tiling of $\mathbf{D}_{j}$ which have valence $v$ and $\alpha$ edges of 
type a. Recall that by hypothesis there are no vertices of valence 1 in the tiling of D. Therefore we can express $V$ as the following sum of the $V(\alpha, v-\alpha)$ 's:

$$
V=\sum_{v=2}^{\infty} \sum_{\alpha=0}^{v} V(\alpha, v-\alpha) \text {. }
$$

Let $E_{\mathrm{a}}$ and $E_{\mathrm{b}}$ denote the number of edges in the tiling of type a and $\mathrm{b}$ respectively, so that $E=E_{\mathrm{a}}+E_{\mathrm{b}}$. Since each a-edge is incident at one vertex, whereas each b-edge is incident at two vertices, we have related sums for $E_{\mathrm{a}}$ and $E_{\mathrm{b}}$ :

$$
E_{\mathrm{a}}=\sum_{v=2}^{\infty} \sum_{\alpha=0}^{v} \alpha V(\alpha, v-\alpha) ; \quad 2 E_{\mathrm{b}}=\sum_{v=2}^{\infty} \sum_{\alpha=0}^{v}(v-\alpha) V(\alpha, v-\alpha) .
$$

Rewriting equation (1) in the form $4 V-2 E_{\mathrm{a}}-2 E_{\mathrm{b}}=4$, we can then combine it with equations (2) and (3) to obtain

$$
\sum_{v=2}^{\infty} \sum_{\alpha=0}^{v}(4-\alpha-v) V(\alpha, v-\alpha)=4
$$

Note that when $v \geq 4$ the coefficient $(4-v-\alpha)$ will be $\leq 0$. We can thus rearrange equation (4) so that all terms on both sides of the equation are nonnegative:

$$
\begin{aligned}
& V(1,1)+2 V(0,2)+V(0,3) \\
&=4+V(2,1)+2 V(3,0)+\sum_{v=4}^{\infty} \sum_{\alpha=0}^{v}(v+\alpha-4) V(\alpha, v-\alpha) .
\end{aligned}
$$

The left-hand side of equation (5) must be at least 4 . Thus there will be a vertex of type $(\mathrm{ab})$ or $(\mathrm{bb})$ or (bbb), according as $V(1,1), V(0,2)$ or $V(0,3)$ is nonzero.

Lemma 8. Let $(\mathbf{D}, \mathbf{H})$ be a good pair. Assume that the tiling of $\mathbf{D}$ has a vertex $V_{0}$ of type $(\mathrm{b}, \mathrm{b}, \mathrm{b})$. Let $P, Q$, and $R$ be the tiles which meet at $V_{0}$. Then there exist a choice of tiles $P$ and $Q$ and a good pair $\left(\mathbf{D}^{\prime}, \mathbf{H}\right)$ with the properties:

(i) $c\left(\mathbf{D}^{\prime}, \mathbf{H}\right)=c(\mathbf{D}, \mathbf{H})$.

(ii) The changes which occur in the passage from $(\mathbf{D}, \mathbf{H})$ to $\left(\mathbf{D}^{\prime}, \mathbf{H}\right)$ do not change the tiling away from $P \cup Q$.

(iii) In the tiling of $\mathbf{D}^{\prime}$ by $\mathbf{H}$ the vertex $V_{0}$ has type (bb).

Proof of Lemma 8. We begin our proof by exhibiting, in Figure 14, the graph of the function $f(x, y)=y^{3}-3 x^{2} y$, in a neighborhood of the origin $(0,0)$. The point $(0,0)$ is a critical point for $f(x, y)$ relative to the height function. That critical point is degenerate, because the Hessian $\left(f_{x y}\right)^{2}-f_{x x} f_{y y}=36\left(x^{2}+y^{2}\right)$ is zero at $(0,0)$. The graph is commonly known as a monkey saddle. Note that there are three "hills" and three "valleys". Level sets are shown in Figure 14. The level sets at $f(x, y)=0$ are the three lines $y=0$ and $y= \pm \sqrt{3}$. Now, we can deform our function a little bit so that the critical point splits into a pair of nondegenerate critical points. For example, let $f_{\varepsilon}(x, y)=y^{3}-3 x^{2} y-3 \varepsilon^{2} y$, which has critical points at $(0, \pm \varepsilon)$. Level sets for the graph of the deformed function $f_{1}$ are shown in Figure 14. By letting $\varepsilon \rightarrow 0$ we conclude that the 

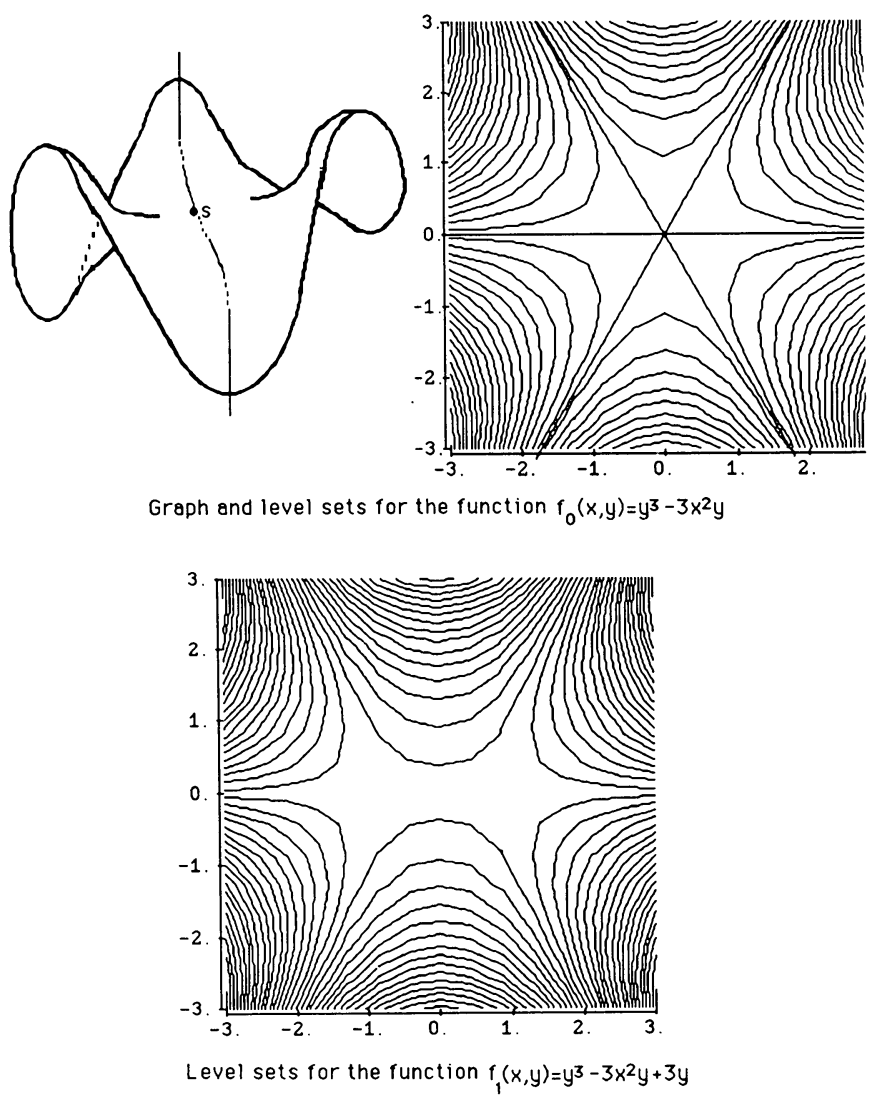

FIGURE 14

orientation of the normal bundle to $f_{\varepsilon}(x, y)$ must be the same at $(0, \varepsilon)$ and at $(0,-\varepsilon)$.

Observe that since the original function $f_{0}(x, y)$ is symmetric under a rotation $g$ of $\pi / 3$ about the origin, this deformation could also have been done so that the "splitting" occurred along the lines $\theta= \pm \pi / 3$ and $\pm 2 \pi / 3$, instead of along the line $\theta=0$, where $\theta$ is the polar angle. Thus there are three ways to do the deformation, defined by the functions $f_{\varepsilon}, g f_{\varepsilon} g^{-1}$, and $g^{2} f_{\varepsilon} g^{-2}$.

We return to $\mathrm{D}$, and to the tiles $P, Q$, and $R$ which meet at $V_{0}$. Two of these three tiles necessarily have the same sign, say $P$ and $Q$. Let $p$ and $q$ be their singular points. The first step is to deform $\mathbf{D}$ so that $p$ and $q$ are close together on $\mathbf{D}$.

Next notice that the foliation of $\mathbf{D}$, together with our knowledge of the signs of the singularities, determines how the portion of $\mathbf{D}$ which is near the singular points is embedded in 3-space. For, choose foliated neighborhoods $\mathbf{N}(p)$ and $\mathbf{N}(q)$ of the singular points. Each is embedded in 3 space as a surface with a single saddle-point singularity. Now band these surfaces together. We may choose the band to be a foliated neighborhood $\mathbf{N}$ of an $\operatorname{arc} \alpha$ on $\mathbf{D}$ which joins $p$ to $q$. There are essentially two ways to embed $\mathbf{N}$ in 3-space, according as the signs of the singularities agree or disagree. See Figure 15. Next arrange that $p$ and $q$ are consecutive singularities in the fibration. These changes can be 

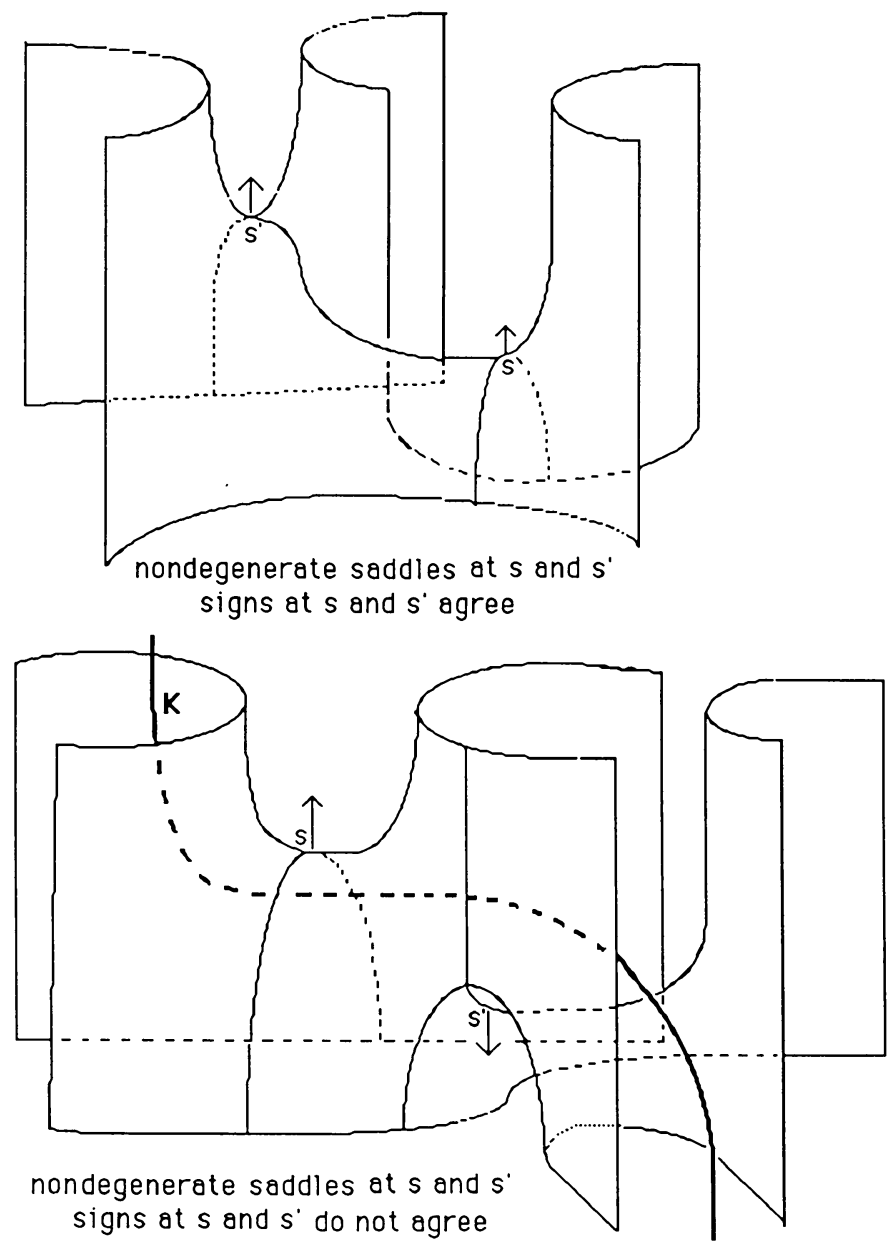

FIGURE 15

done so that the tiling is unchanged away from $P \cup Q$. A further deformation ensures that in some neighborhood of the deformed critical points the surface is the graph of $f_{1}$. (This is the place where we use the fact that the signs of the singularities agree.) We can then let $\varepsilon \rightarrow 0$, so that the embedding changes to the graph of $f_{0}$. From Figure 15 it is clear that the link $\mathbf{K}$ and other sheets of $\mathbf{D}$ will not obstruct this deformation (if the signs disagree they might, as is illustrated in the bottom picture in Figure 15). Finally we can introduce a new deformation in the fibration to achieve the graph of $g f_{1} g^{-1}$.

We now examine the effect of the passage from $f_{1}$ to $g f_{1} g^{-1}$ on the tiling of D. First note that without loss of generality we may assume that our changes were supported in a neighborhood in $S^{3}$ of $P \cup Q$. There are three possibilities for the types of $P$ and $Q$, i.e. both could be type bb, or $P$ might be type bb and $Q$ type ab, or both could be type ab. In all three cases the tiles $P$ and $Q$ have a common edge which has type $\mathbf{b}$, and $V_{0}$ is at one of the endpoints of this edge. We show "before" and "after" tilings for all three cases in Figure 16. In every case $V_{0}$ has been changed from a vertex of type (bbb) to one of type (bb), as required. 

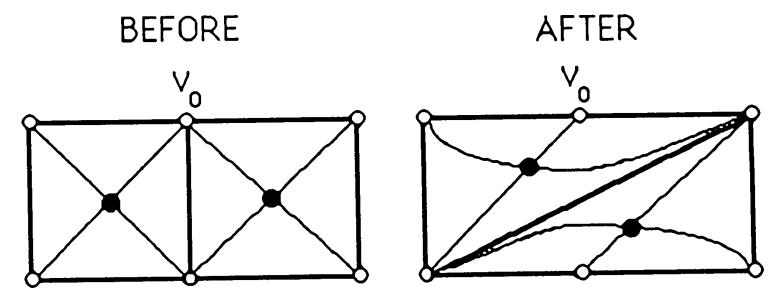

Types (bb) and (bb)
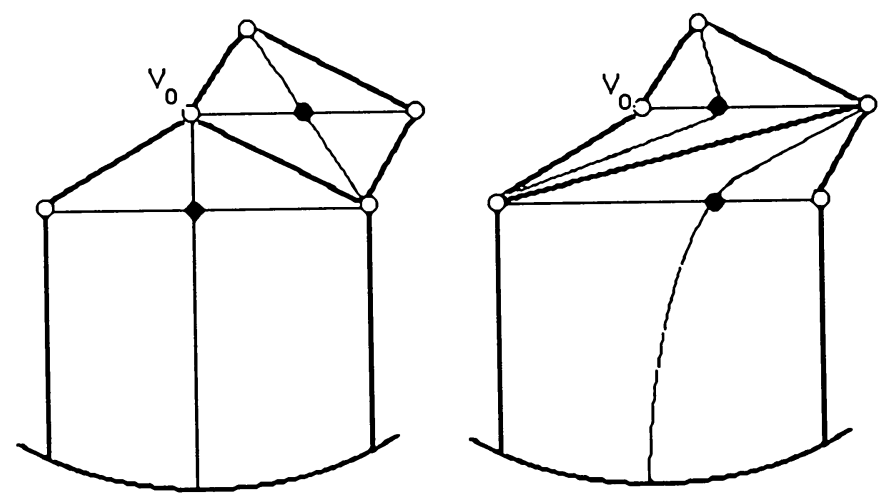

Types (bb) and (ab)

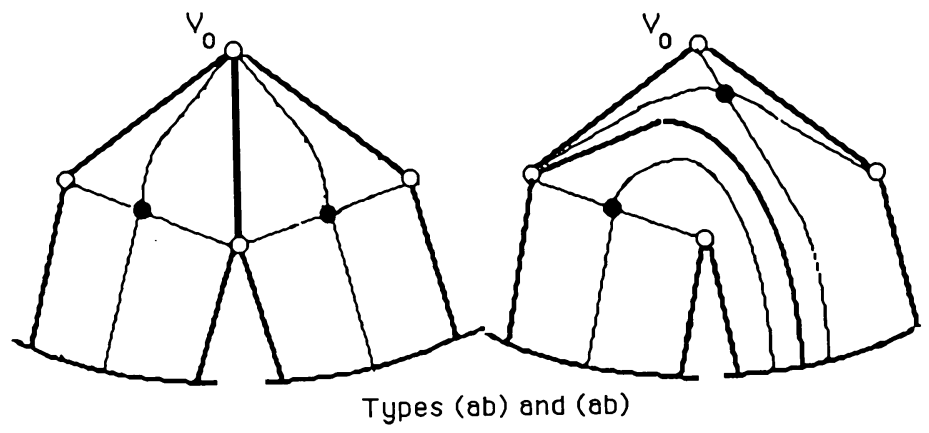

FIGURE 16

Lemma 9. Let $(\mathbf{D}, \mathbf{H})$ be a good pair. Suppose that the $\mathbf{H}$-tiling of $\mathbf{D}$ has a vertex of type $(\mathbf{a}, \mathbf{b})$ with sign $( \pm, \pm)$. Then there exists a good pair $\left(\mathbf{D}^{\prime}, \mathbf{H}\right)$, with $c\left(\mathbf{D}^{\prime}, \mathbf{H}\right)=c(\mathbf{D}, \mathbf{H})$, such that the tiling of $\mathbf{D}^{\prime}$ has a vertex of type $(\mathbf{a})$.

Proof of Lemma 9. A vertex $V_{1}$ of type (ab) can only occur when the tiling of a component $\mathbf{D}_{i}$ of $\mathbf{D}$ has two type ab-tiles $P$ and $Q$ which are identified along common $\mathbf{a}$ and $\mathbf{b}$ edges, so that the two tiles also have two vertices, $V_{0}$ and $V_{1}$, in common, as in Figure 17. By hypothesis, our tiles have the same sign, so we are in exactly the situation of Lemma 8 , with $P$ and $Q$ both type ab-tiles. We construct $\left(\mathbf{D}^{\prime}, \mathbf{H}^{\prime}\right)$ as in the proof of Lemma 8, to produce a new tiling, as illustrated in the right picture in Figure 17, which is essentially identical with the corresponding picture in Figure 16. (The only difference is that in the situation of Figure 16 there could be other tiles glued to $P$ and $Q$ at the vertex $V_{1}$, so 

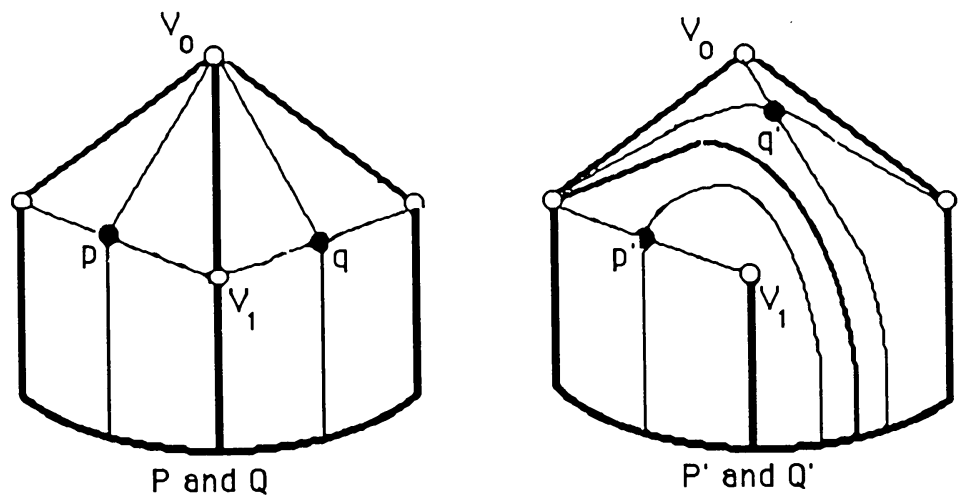

FIGURE 17

that the picture in Figure 16 is less restrictive than that in Figure 17.) After the change we obtain an aa-tile, with a vertex $V_{1}$ of type (a).

\section{Closed bRaId REPRESENTATIVES OF THE UNLINK}

Theorem 1 and Corollary 2 were stated in the introduction. In this section we use the machinery of $\S 2-5$ to prove them. Theorem 1 is our version of Markov's Theorem without stabilization, for the unlink.

Proof of Theorem 1. We are given a closed $n$-braid representative $\mathbf{K}$ of the $r$-component unlink, with braid axis $\mathbf{A}$. We want to show that we can reduce it to the standard $r$-braid representative by isotopy in the complement of the axis, removal of trivial loops, and exchanges. By our work in $\S 2$, we may assume that there is a good pair $(\mathbf{D}, \mathbf{H})$, where $\partial \mathbf{D}=\mathbf{K}, \partial \mathbf{H}=\mathbf{A}$. The complexity of this pair is $c(\mathbf{D}, \mathbf{H})$. The proof will be by induction on $c(\mathbf{D}, \mathbf{H})$.

Our first assertion is that if $c(\mathbf{D}, \mathbf{H})=(r, 0)$, then $\mathbf{K}$ is the standard $r$-braid representative $\mathbf{U}_{r}$ of the $r$-component unlink. To see this, note that there are $r$ components in $\mathbf{D}$, and each is pierced at least once by $\mathbf{A}$, because $\mathbf{K}$ is a closed braid with respect to $\mathbf{A}$. By the definition of $c(\mathbf{D}, \mathbf{H})$ the axis $\mathbf{A}$ pierces the disc family $\mathbf{D}$ in exactly $r$ points. Thus each component $\mathbf{D}_{i}$ is pierced once by $\mathbf{A}$. Since $(\mathbf{D}, \mathbf{H})$ is a good pair the foliation of $\mathbf{D}$ is the standard radial foliation near each point of $\mathbf{A} \cap \mathbf{D}$. From this it follows that each component of $\mathbf{D}$ is foliated without singularities by arcs with one endpoint on $\mathbf{A}$ and the other on $\mathbf{K}$. The foliation of $\mathbf{D}_{i}$ will therefore be standard on all of $\mathbf{D}_{i}$. Thus $\partial \mathbf{D}_{i}$ is a standard 1-braid for each $i=1, \ldots, r$ and so $\mathbf{K}=\mathbf{U}_{r}$.

In view of this, we assume from now on that $c(\mathbf{D}, \mathbf{H})>(r, 0)$. We must show that, after isotopy in the complement of the axis, deletion of trivial loops, and exchange moves we can reduce the complexity.

We examine the tiling of $\mathbf{D}$ which is determined by $\mathbf{H}$. If there is a vertex of type (a), then by Lemma 4 we know we can reduce complexity by eliminating a trivial loop. We can therefore assume that there are no vertices of valence 1 in the tiling of $\mathbf{D}$. It then follows from Lemma 7 that any component $\mathbf{D}_{i}$ which is nontrivially tiled must have a vertex of type (ab) or (bb) or (bbb). By Lemma 8 , we can do better: we can assume there is a vertex of type $(a b)$ or $(b b)$. If there is a vertex of type $(a b)$ and sign $(++)$ or $(--)$, then by Lemma 9 we can change the fibration to produce a trivial loop, and again we are done. If there 
exists a vertex of type $(a b)$ with sign $(+,-)$, then by Lemma 5 we can reduce the complexity by an exchange move, and again we are done. If there is a vertex of type (bb) then by Lemma 5 an exchange move reduces the complexity. The proof of Theorem 1 is complete.

Corollary 2 asserts that the stabilization index of an arbitrary braid representative of the $r$-component unlink is 1 .

Proof of Corollary 2. Let $\mathbf{K}$ be an arbitrary $n$-braid representative of the unlink $\boldsymbol{U}_{r}$, and let $\boldsymbol{U}_{r}$ be the standard $r$-braid representative. Consider any chain of braids which takes $\mathbf{K}$ to $\mathbf{U}_{r}$ and has the property that the chain contains braids of index $n^{\prime}>n$. Then there is a braid $\mathbf{K}^{\prime}$ of braid index $n$ in the chain, and a subchain from $\mathbf{K}$ to $\mathbf{K}^{\prime}$ which contains a braid of index $n^{\prime}$. By Theorem 1, the subchain may be replaced by a sequence of braids, all in $B_{n}$ and related to one another by exchanges and conjugations in $B_{n}$, and since an exchange on weighted strands can be replaced by a sequence of exchanges which involve only strands with weight 1 , we can assume that all the weights are 1 . So, it only remains to show that a single exchange in $B_{n}$ which involves strands of weight 1 can be replaced by a chain in which the intermediate braids have braid index at most $n+1$. However, this was proved in 1938, by Weinberg, who gave in [W] a very simple replacement sequence. Weinberg's sequence shows that an exchange can be realized by adding one trivial loop, conjugating, and then deleting a trivial loop.

Comments. The earliest version of Markov's theorem was the announcement [Ma], which described the theorem and sketched an outline of a proof. That version was a little bit different from the version we have given here, as it included the exchange move in addition to (Mi) and (Mii). A short time after Markov's announcement was published Weinberg [W] showed (using the replacement sequence we have just described) that the exchange move was a consequence of (Mi) and (Mii). It was thus removed from subsequent versions of what has come to be known as "Markov's Theorem".

The main result of this paper is a type of converse to Weinberg's result, vis: if $\mathbf{K}$ represents the unlink, then the addition and subsequent deletion of trivial loops can be replaced by conjugation and exchanges. In a subsequent manuscript we will prove a version of Markov's theorem without stabilization for an arbitrary link $K$. As will be seen, the exchange move is not enough in the general case; one needs a generalization of it which we call a "braidpreserving flype". Corollary 2 will be seen to generalize as well, to show that $S(K, n)=2 n$.

\section{ACKNOWLEDGMENTS}

We thank M. Boileau, E. Flapan, B. Wajnryb, Y. Moriah, X. Q. Wu, S. Schanuel, D. Schack, and X. S. Lin for helpful comments and discussions.

Special thanks go to Melissa Menasco and to Joseph Birman, for their support and encouragement during many visits of Birman to the Menasco home and Menasco to the Birman home. This work, which is part of a much larger study still in progress, could not have been completed without their cooperation. 


\section{REFERENCES}

[A] J. W. Alexander, A lemma on systems of knotted curves, Proc. Nat. Acad. Sci. U.S.A. 9 (1923), 93-95.

[Be] Daniel Bennequin, Entrelacements et equations de Pfaff, Asterisque 107-108 (1983), 87161.

[Bi] Joan S. Birman, Braids, links and mapping class groups, Ann. of Math. Stud., no. 82, Princeton Univ. Press, Princeton, N.J., 1974.

[B-M,I] Joan S. Birman and William W. Menasco, Studying links via closed braids. I: A finiteness theorem, Pacific J. Math. (to appear).

[B-M,II] __ Studying links via closed braids. II: On a theorem of Bennequin, Topology Appl. (to appear).

[B-M,III] __ Studying links via closed braids. III: Classifying links which are closed 3-braids, preprint, 1989.

[B-M,IV] _ Studying links via closed braids. IV: Split and composite links, Invent. Math. 102 Fasc. 1 (1990), 115-139.

[B-M,VI] __ Studying links via closed braids. VI: A non-finiteness theorem, Pacific J. Math. (to appear).

[J] V. Jones, Hecke algebra representations of braid groups and link polynomials, Ann. of Math. 126 (1987), 335-388.

[G] F. Garside, The braid groups and other groups, Quart. J. Math. Oxford 20 235-254.

[Ma] A. A. Markov, Uber die freie Aquivalenz der geschlossenen Zopfe, Rec. Soc. Math. Moscou 43 (1936), 73-78.

[Mo,1] Hugh R. Morton, An irreducible 4-string braid with unknotted closure, Math. Proc. Cambridge Philos. Soc. 93 (1983), 259-261.

[Mo,2] __ Threading knot diagrams, Math. Proc. Cambridge Philos. Soc. 99 (1986), 247-260.

[R] L. Rudolph, Braided surfaces and Seifert ribbons for closed braids, Comment. Math. Helv. 58 (1983), 1-37.

[W] N. Weinberg, Sur l'equivalence libre des tresses fermees, Dokl. Akad. Sci. USSR 23 (1939), no. 3 .

Department of Mathematics, Columbia University, New York, New York 10027

Department of Mathematics, SUNY at Buffalo, Buffalo, New York 14222 